\title{
Development of multiple visceral artery pseudoaneurysms following pancreatic injury from penetrating trauma
}

\author{
Harold J. Leraas ${ }^{1 *}$, Brian F. Gilmore ${ }^{1}$, Uttara P. Nag ${ }^{1}$, Megan C. Turner ${ }^{1}$, Ryan S. Turley ${ }^{1}$ and Mitchell W. Cox ${ }^{1}$ \\ ${ }^{1}$ Department of Surgery Department of Surgery, Duke University, USA
}

\begin{abstract}
Visceral artery pseudoaneurysm is a rare, life-threatening pathology, which can lead to life threatening intrabdominal hemorrhage if left untreated. Diagnosis is often difficult due to vague symptomology. Herein, we present a case of a patient presenting with multiple delayed visceral arterial pseudoaneurysms due to a pancreatic leak following an exploratory laparotomy for several abdominal gunshot wounds. Open intervention was considered prohibitively high risk, given the patient's hostile abdomen and loss of normal tissue planes secondary to inflammation. Due to the complexity of the anatomy involved in these pseudoaneurysms, this was managed successfully with a combination of coil embolization and stent-graft placement.
\end{abstract}

\section{Introduction}

Visceral artery pseudoaneurysm (VAPA) is a life-threatening pathology, with annual incidence of $0.1-2.0 \%$ [1]. VAPAs, due to a combination of rarity and vague symptoms, are frequently unrecognized until a catastrophic hemorrhage; mortality rates from ruptured visceral arterial aneurysms as high as $36 \%$ have been described [2].

VAPA can occur following instrumentation of the abdomen or blunt abdominal injury [3,4]. A subset of VAPAs occurs after pancreatic injury due to pancreatic leak and subsequent enzymatic degradation of neighboring vasculature [5].

Herein, we present a case of delayed VAPA two weeks after sustaining a pancreatic injury due to abdominal gunshot wounds. Due to the patient's known prolonged pancreatic leak, open surgical approaches were thought to carry an unacceptable risk of inadvertent visceral injury. Although most documented cases of VAPAs are singular, this case presented unique challenges as multiple VAPAs required intervention. We instead pursued an aggressive endovascular approach, managing one VAPA with embolization and a second with stent-grafting.

\section{Case report}

A 34-year-old male presented to the emergency department via ambulance following two abdominal gunshot wounds. His medical history consisted of multiple traumatic injuries including two stab wounds and four admissions for blunt trauma. He was noted by prehospital personnel to have a GCS of 15, however a blood pressure was unable to be recorded. Upon presentation to the hospital he was found to have a heart rate in the 140s with palpable femoral pulses but absent pedal pulses, and a GCS of 12 . He was taken emergently to the operating room for an exploratory laparotomy due to his penetrating abdominal wounds and hemodynamic lability.

Intra-operatively, he was found to have lacerations of the liver and pancreatic head, three proximal jejunal enterotomies, a partial thickness descending colon injury, mesenteric bleeding, and a gallbladder injury. Ultimately the patient underwent cholecystectomy, cauterization of liver lacerations, primary repair of the enterotomies and the colon injury, as well as wide drainage of the pancreatic head injury with two Jackson-Pratt drains. Postoperatively the patient was transferred to the surgical intensive care unit.

A combination of a prolonged ileus and persistent leukocytosis prompted a CT scan on post-operative day 7 which demonstrated both a superior mesenteric vein thrombosis as well as portal venous gas and a perihepatic fluid collection consistent with a missed duodenal injury. This was managed with percutaneous drainage of the fluid collection due to the patient's stability. He remained stable but critically ill until post-operative day 18 , at which point he became acutely hypotensive and tachycardic with a drop in hemoglobin levels. A CT angiogram was performed which demonstrated two VAPAs, one measuring $19 \times 15 \mathrm{~mm}$ in the mid-superior mesenteric artery (SMA) and a second measuring $10 \times 9 \mathrm{~mm}$ in the distal SMA. The smaller of these two began just distal to an occlusion of the SMA. In light of the significant intra-abdominal contamination and extensive injuries, the patient was deemed a poor candidate for an open approach.

The patient was taken to a hybrid operating room where vascular access was obtained via the left brachial artery. An arteriogram (Figure 1) demonstrated the expected two pseudoaneurysms in the SMA with active extravasation. The point of occlusion at the origin of the distal VAPA was traversed with a combination of a 0.035 " glidewire and Quick-cross catheter (Spectranetics). Luminal re-entry was confirmed and a $6 \times 22 \mathrm{~mm}$ ICast (Atrium) covered balloon expandable stent was deployed, excluding the pseudoaneurysm and restoring in-line SMA flow while sacrificing only a single jejunal branch (Figure 2). The proximal VAPA was embolized using a 0.021" Renegade catheter and Interlock coil system (Boston Scientific) with $4 \mathrm{~mm}$ and $5 \mathrm{~mm}$ diameter

Correspondence to: Harold J. Leraas, Duke University Medical Center, Durham, NC 27710. Tel: (360) 888-3643; Fax: (919) 681-7934; E-mail: harold.leraas@ dm.duke.edu

Key words: visceral artery pseudoanurysm, pancreatic trauma, vascular surgery

Received: April 03, 2017; Accepted: May 02, 2017; Published: May 05, 2017 


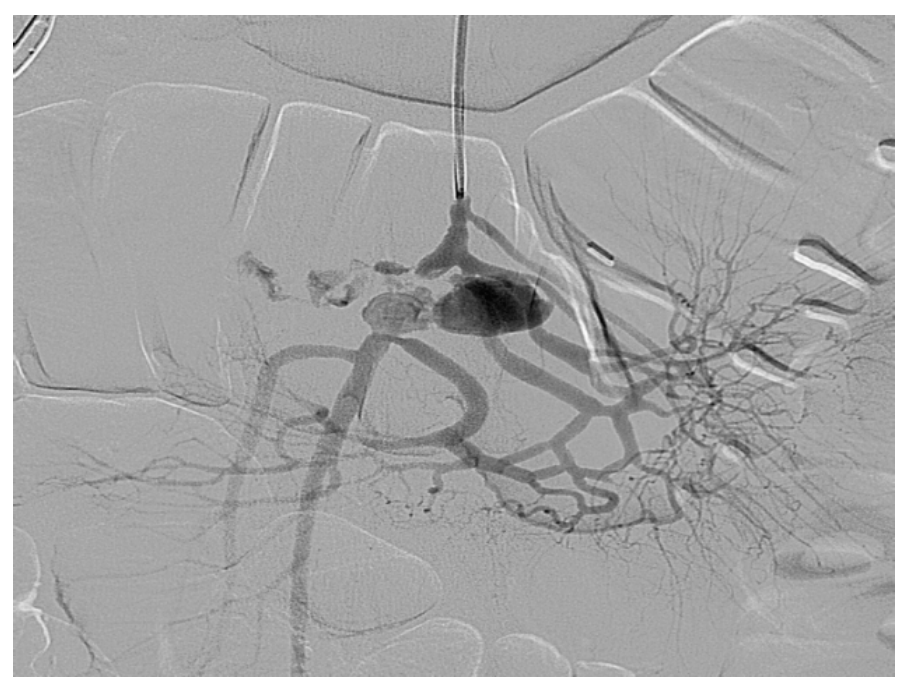

Figure 1. Initial Diagnostic Arteriography of SMA injury.

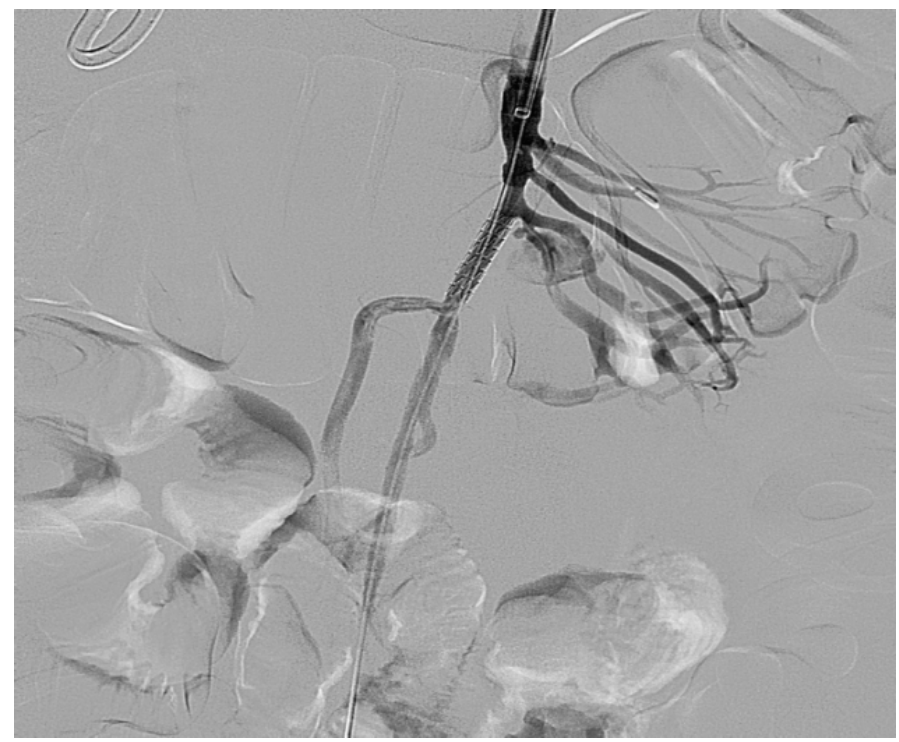

Figure 2. Arteriography demonstrating stent placement, $1.0 \times 0.9 \mathrm{~cm}$ VAPA resolution, and occlusion of arterial branch.

interlock-18 Coils were deployed in the outflow vessel of the VAPA and an additional six $10 \mathrm{~mm}$ and $12 \mathrm{~mm}$ interlock-18 coils placed into the pseudoaneurysm itself (Figure 3). Collateral intestinal blood flow and successful exclusion of the pseudoaneurysms were documented on completion arteriogram (Figure 4).

The patient ultimately recovered well with no additional interventions required. He was discharged on 7 days after endovascular intervention on a regimen of clopidogrel ( $75 \mathrm{mg}$ daily) for his stent and rivaroxaban (20 mg daily) for his SMV thrombosis.

\section{Discussion}

Standard of care for VAPA is largely a matter of opinion. Surgical ligation was once the preferred treatment, however open and endovascular approaches show similar mortality rates [6]. While many still advocate for open isolation and resection of VAPAs, coil embolization and stenting are safe alternatives for patients deemed unsuitable for open surgery [7-9].
In this case, repeat laparotomy and retroperitoneal dissection were deemed treacherous due to extensive adhesions, loss of tissue planes, and persistent abdominal contamination necessitating endovascular intervention. Interestingly, combinations of endovascular approaches were required for correction of multiple VAPAs. His case demonstrates several possible etiologies for VAPAs, including unrecognized injury from penetrating trauma, instrumentation of the abdomen during exploration, or sequelae of pancreatic injury and leak. This patient's VAPAs were not present during the initial exploratory laparotomy, therefore we suspect that the pancreatic injury sustained in the initial trauma resulted in continued leak, leading to development of VAPAs.

The majority of current the literature surrounding VAPAs consists of small case series and case reports. While this existing data suggests that endovascular intervention is increasingly being used to manage VAPAs when laparotomy is considered prohibitively dangerous, there is a relative paucity of reports documenting successful endovascular management of extremely complicated or multiple VAPAs. Even

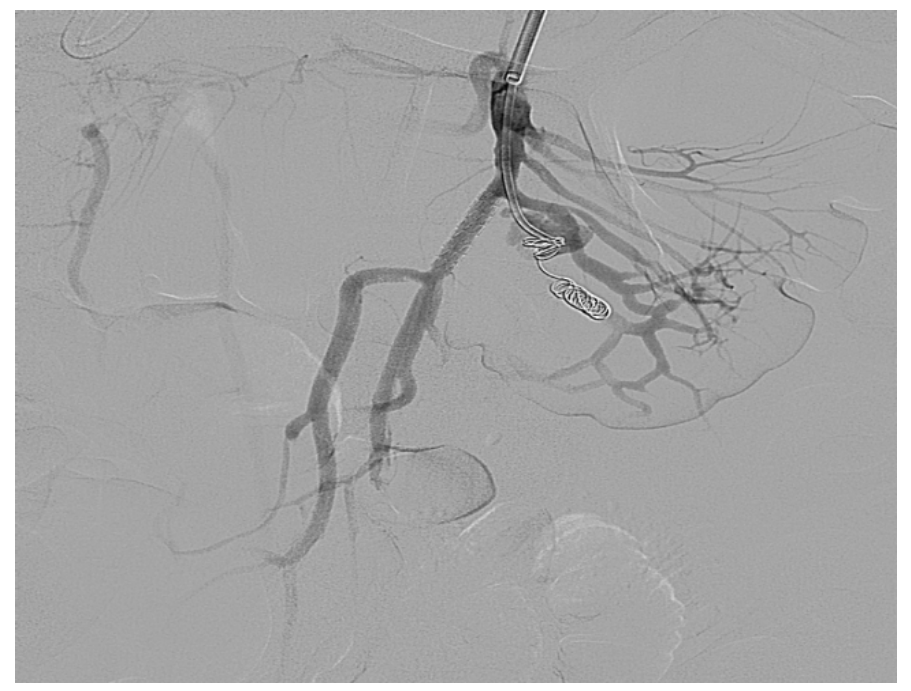

Figure 3. Arteriography demonstrating placement of coil to resolve $1.9 \mathrm{~cm} \times 0.5 \mathrm{~cm}$ VAPA

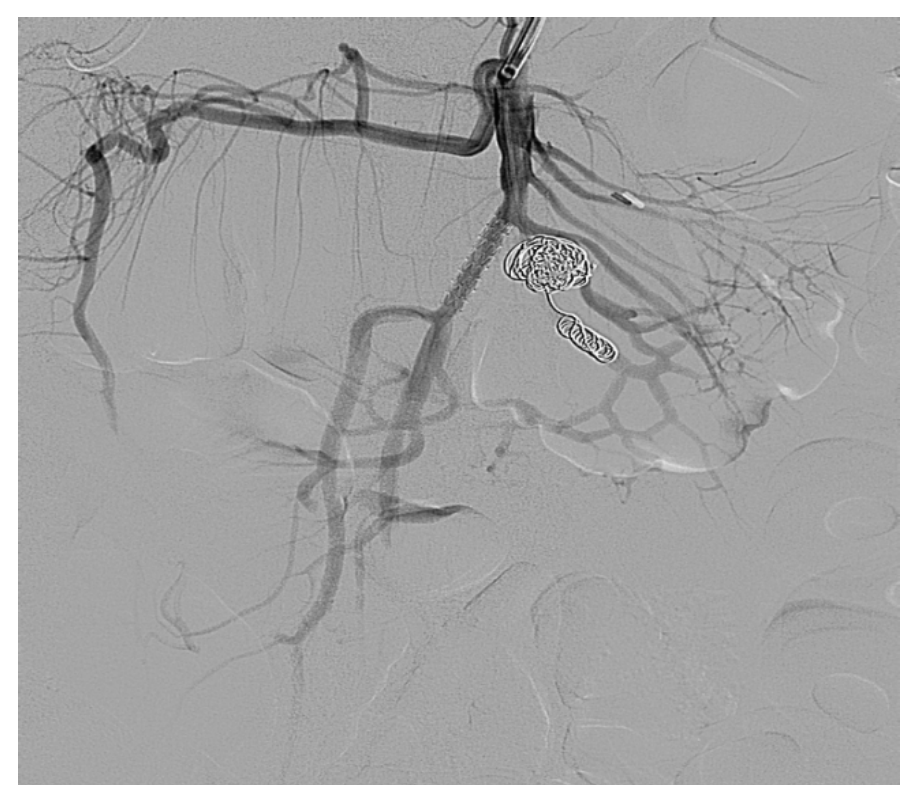

Figure 4. Arteriography demonstrating completed stent and coil embolization. Appropriate collateral flow to the intestines is visible through collateral arteries. 
though both embolization and stenting have been employed previously, our case demonstrates that these techniques can be used in combination for the management of even anatomically complex lesions.

\section{Financial disclosures}

None

\section{References}

1. Fankhauser GT, Stone WM, Naidu SG, Oderich GS, Ricotta JJ, et al. (2011) The minimally invasive management of visceral artery aneurysms and pseudoaneurysms. J Vasc Surg 53: 966-970. [Crossref]

2. Chadha M, Ahuja C (2009) Visceral Artery Aneurysms: Diagnosis and Percutaneous Management. Semin Intervent Radiol 26: 196-206. [Crossref]

3. Etezadi V, Gandhi RT, Benenati JF, Rochon P, Gordon M, et al. (2011) Endovascular treatment of visceral and renal artery aneurysms. J Vasc Interv Radiol 22: 1246-1253. [Crossref]
4. Nagarajan K, Elango S, Ishita L, Shanmugam D (2016) Traumatic Pseudoaneurysm of the Inferior Mesenteric Artery Branch: A Rare Cause of Lower GI Bleeding and Treatment with Selective Coil Embolization. Cardiovasc Intervent Radiol 39: 1358-1360.

5. Ding XJ, Zhu JK, Zhu M (2011) Therapeutic Management of Hemorrhage from Visceral Artery Pseudoaneurysms after Pancreatic Surgery. J Gastrointes Surg 15: $1417-1425$.

6. Nayara Cioffi Batagini HE-A, Daniel G, Clair, Lee Kirksey (2016) Open versus Endovascular Treatment of Visceral Artery Aneurysms and Pseudoaneurysms. Annals of Vasc Surg.

7. Pitton MB, Dappa E, Jungmann F, Kloeckner R, Schotten S, et al. (2015) Visceral artery aneurysms: Incidence, management, and outcome analysis in a tertiary care center over one decade. Eur Radiol 25: 2004-2014. [Crossref]

8. Kalva SP, Yeddula K, Wicky S, Fernandez del Castillo C, Warshaw AL (2011) Angiographic intervention in patients with a suspected visceral artery pseudoaneurysm complicating pancreatitis and pancreatic surgery. Arch Surg 146: 647-652. [Crossref]

9. Marone EM, Mascia D, Kahlberg A, Brioschi C, Tshomba Y, et al. (2011) Is open repair still the gold standard in visceral artery aneurysm management? Ann Vasc Surg 25: 936-946. [Crossref]

Copyright: $\bigcirc 2017$ Leraas HJ. This is an open-access article distributed under the terms of the Creative Commons Attribution License, which permits unrestricted use, distribution, and reproduction in any medium, provided the original author and source are credited. 